\title{
Amino acids other than glutamate affect the expression of the GAD system in Listeria monocytogenes enhancing acid resistance
}

\section{Article}

Accepted Version

Creative Commons: Attribution-Noncommercial-No Derivative Works 4.0

Paudyal, R., O'Byrne, C. P. and Karatzas, K.-A. (2020) Amino acids other than glutamate affect the expression of the GAD system in Listeria monocytogenes enhancing acid resistance. Food Microbiology, 90. 103481. ISSN 0740-0020 doi: https://doi.org/10.1016/j.fm.2020.103481 Available at https://centaur.reading.ac.uk/92900/

It is advisable to refer to the publisher's version if you intend to cite from the work. See Guidance on citing.

Published version at: https://www.sciencedirect.com/science/article/pii/S0740002020300708

To link to this article DOI: http://dx.doi.org/10.1016/j.fm.2020.103481

Publisher: Elsevier

All outputs in CentAUR are protected by Intellectual Property Rights law, including copyright law. Copyright and IPR is retained by the creators or other copyright holders. Terms and conditions for use of this material are defined in the End User Agreement. 


\section{CentAUR}

Central Archive at the University of Reading

Reading's research outputs online 

monocytogenes enhancing acid resistance

3

4 Ranju Paudyal ${ }^{1}$, Conor P. O'Byrne ${ }^{2}$ and Kimon-Andreas Karatzas ${ }^{1 *}$

$5{ }^{1}$ Department of Food and Nutritional Sciences, The University of Reading, PO Box 226,

6 Whiteknights, Reading RG6 6AP, UK.

${ }^{2}$ Bacterial Stress Response Group, Microbiology, School of Natural Sciences, National

University of Ireland, Galway, Galway, Ireland

10

11

$12 *$ Corresponding author

13 Email address: k.karatzas@reading.ac.uk

14 Corresponding author address:

15 School of Chemistry, Food and Pharmacy

16

Department of Food \& Nutritional Sciences,

University of Reading,

18

Reading

19 RG6 6AD

UK

22

Tel. +44 1183786678

23

Fax. +44 1189310080

24

Email: k.karatzas@reading.ac.uk

25

26 


\section{ABSTRACT}

\section{$47 \quad$ Keywords}

49 and other microorganisms under acidic conditions. Environmental conditions influence the function of the GAD system. Until now, the only conditions known to lead to increased transcription of the GAD system are the stationary phase in rich media and anoxic conditions. Previously, we showed that transcription of the GAD system requires unidentified compounds other than glutamate present in rich media. Following a test looking at various compounds we identified for first time that peptone, tryptone and casamino acids activate the GAD system under oxic conditions suggesting that amino acid(s) other than glutamate and/or peptides are important for the above process. The defined medium, where the GAD system is inactive, once it is supplemented with the above compounds results in an active intracellular and extracellular GAD system and increased acid resistance. Through functional genomics we show that these compounds are required for GadD2 activity and although we previously showed that GadD3 is active part of the intracellular GAD system, the supplementation did not activate this gene. The above is explained by the fact that only gadD2 transcription was upregulated by these compounds while the transcription of gadD1 and gadD3 remained unaffected. Together our results show that the L. monocytogenes GadD2 decarboxylase is activated in the presence of amino acids or peptides other than glutamate, a finding that has important implications for acid tolerance and food safety.

Glutamate decarboxylase_system; L. monocytogenes; peptone; tryptone; casaminoacids; $\gamma$-aminobutyrate; acid tolerance; GABA 
The Glutamate Decarboxylase System (GAD) system is present in a wide variety of living organisms from all kingdoms of life, playing different roles ranging from brain functions in mammals, to response under stress conditions such as temperature shock and hypoxia in plants (Shelp et al., 1999) and to acid resistance in microorganisms. The main role of the GAD system in microorganisms is protection from acidic conditions (Capitani et al., 2003; Cotter et al., 2001a; Su et al., 2011) and in some of them such as L. monocytogenes (Cotter et al., 2001a) and Escherichia coli (Capitani et al., 2003; Foster 2004) it is the most important acid resistance mechanism. The GAD system is present in many other bacteria such as Shigella flexneri (Waterman and Small, 2003), Mycobacterium tuberculosis (Cole et al., 1998; Cotter et al., 2001a), Lactobacillus reuteri, Lactococcus lactis, Lactobacillus plantarum (Su et al., 2011) and Brucella spp. (Damiano et al. 2014). L. monocytogenes normally possesses three decarboxylases GadD1, GadD2 and GadD3 and two antiporters GadT1 and GadT2 (Cotter et al., 2005; Karatzas et al., 2012). The five corresponding genes are arranged in three separate operons namely gadD1T1, gadT2D2 and gadD3 (Cotter et al., 2005). The gadD1T1 operon has been shown to enhance growth under mild acidic conditions (Cotter et al., 2005), while gadD2T2 plays important role in survival under severe acidic conditions (Cotter et al., 2001a; Cotter et al., 2005). Furthermore, we have shown that the GAD system comprises two distinct parts i) the extracellular glutamate decarboxylase system $\left(\mathrm{GAD}_{\mathrm{e}}\right)$ and ii) the intracellular glutamate decarboxylase $\left(\mathrm{GAD}_{\mathrm{i}}\right)$ system (Karatzas et al., 2012). The $\mathrm{GAD}_{\mathrm{e}}$ is the part of the GAD system that imports glutamate from the extracellular environment through the antiporters and the decarboxylases convert it to $\gamma$-amino butyric acid (GABA) and $\mathrm{CO}_{2}$. This reaction consumes a proton resulting in an increase of intracellular $\mathrm{pH}$. Subsequently, the GABA is 
exported via the antiporters GadT in exchange for a further glutamate molecule. The GADi has previously been described converting intracellular pools of glutamate to intracellular GABA $\left(\mathrm{GABA}_{i}\right.$; (Karatzas et al., 2010; Karatzas et al., 2012). Subsequently, GABA $\mathrm{i}_{\mathrm{i}}$ can directed through the GABA shunt and converted to succinate semi aldehyde and succinate (Feehily et al., 2013). Most strains of L. monocytogenes such as $10403 \mathrm{~S}$ and LO28 utilize both $\mathrm{GAD}_{\mathrm{i}}$ and $\mathrm{GAD}_{\mathrm{e}}$ (Karatzas et al., 2010) whereas EGD-e which is the most studied L. monocytogenes strain, utilizes only the $\mathrm{GAD}_{\mathrm{i}}$ system resulting in a highly acid sensitive phenotype (Feehily et al., 2013).

It is well known that the GAD system is expressed in rich and complex media like Brain Heart Infusion (BHI; Hersh et al., 1996; Penfound et al., 1999). However, we have shown that the $L$. monocytogenes GAD system is not being upregulated under acidic conditions, as somebody would expect and its expression depends on unknown signalling compounds present in rich growth media. To date, little is known about the regulation and expression of the GAD system in L. monocytogenes. GadD2, the most potent part of the GAD system is known to be upregulated under anoxic conditions (Feehily et al., 2013; Jydegaard-Axelsen et al., 2004), during stationary phase (Cotter et al., 2001b) and in gastric fluid (Cotter et al., 2001a) while we are not aware of any other conditions leading to its upregulation. L. monocytogenes requires specific media for growth and for the GAD system to be expressed (Cotter et al., 2001b; Penfound et al., 1999). The inability of the GAD system to be expressed in a defined medium (DM) supplemented with glutamate (Glt; DMG) has been previously shown by Karatzas et al., (2010). In the same study, it was shown that the transcription of the GAD genes was minimal in a defined medium supplemented with glutamate (DMG; Karatzas et al., 2010). Therefore, this medium could be used to study environmental conditions and compounds that trigger the expression of the GAD system, in order to gain insights into how this important acid resistance mechanism is regulated in L. monocytogenes. 
The main aim of this study was to identify compounds that are essential for the expression and function of the GAD system through the use of DMG medium as a basis. During these experiments a number of compounds and mixtures of components were tested and tryptone, peptone and casamino acids were identified as activators of the GAD system. Furthermore, we identified the impact of these mixtures of compounds on survival under acidic conditions, on the $\mathrm{GAD}_{\mathrm{e}}$ and $\mathrm{GAD}_{\mathrm{e}}$ system activity, the role of each one of the GAD genes and on transcription of the GAD genes.

Knowledge of the environmental conditions that activate or inhibit the GAD system can help to understand the acid resistance of this pathogen since the GAD system is the most important mechanism of acid resistance. This knowledge could help us predict the survival of $L$. monocytogenes in various acidic foods or environments or even help us manipulate its acid resistance through interventions that could affect the availability of these activators. These findings could have a major economic impact on the food industry and lead to new methods reducing the incidence of listeriosis whose prevalence in the EU and UK has increase significantly the last years (EFSA and ECDC, 201). Furthermore, since $L$. monocytogenes is a model microorganism for the study of the GAD system in Gram-positive bacteria, that is distinctly different from that of Gram-negative bacteria such as $E$. coli, it could contribute to the wider understanding of acid resistance in Gram-positive organisms.

\section{MATERIALS AND METHODS}

\section{Bacterial strains and growth conditions}

Mutants in the three GAD decarboxylases of $10403 \mathrm{~S}$ and their isogenic WT were used in this study (Table 1). All strains were stored at $-80^{\circ} \mathrm{C}$ in cryovial tubes with $7 \%$ DMSO. Stock 
cultures from $-80^{\circ} \mathrm{C}$ were grown in BHI Agar (LAB M, Lancashire, UK) and plates incubated at $37^{\circ} \mathrm{C}$ overnight. Three colonies from each plate were transferred into $3 \mathrm{ml}$ of sterile BHI (LAB M, Lancashire, UK) and incubated overnight at $37^{\circ} \mathrm{C}$ with shaking at $140 \mathrm{rpm}$. DMG (Amezaga et al., 1995) was prepared by supplementation of $6.8 \%$ of either tryptone (Thermo Scientific Oxoid, LP0042, Basingstoke, UK), peptone (Thermo Scientific Oxoid, CM009, Basingstoke, UK) and casamino acids (Difco, Michigan, USA). Similarly, for other experiments DMG was prepared with $3.6 \%$ peptone or $10 \%$ casamino acids. Subsequently, the overnight cultures were used to inoculate $20 \mathrm{ml}$ of sterile DMG medium ( $1 \%$ inoculum) either with or without supplementation in $250 \mathrm{ml}$ conical flasks and incubated at $37^{\circ} \mathrm{C}$ with shaking at $140 \mathrm{rpm}$ overnight $(\sim 24 \mathrm{~h})$. Subsequently, these overnight cultures were used in all other experiments. As described below in GABase Assay, GABAe was measured in DMG under

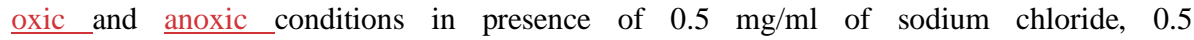
$\mathrm{mg} / \mathrm{ml}$ maleic acid, $0.5 \mathrm{mg} / \mathrm{ml}$ succinic acid, $0.5 \mathrm{mg} / \mathrm{ml}$ acetic acid, $0.5 \mathrm{mg} / \mathrm{ml}$ lactic acid, $0.1 \%$ sodium deoxycholate, $5 \%$ oxgall, $5 \%$ bile salts, $2 \%, 10 \%$ casamino acids, $3.6 \%$ peptone and $6.8 \%$ tryptone was also used to measure $\mathrm{GABA}_{\mathrm{e}}$

\section{Survival under acidic conditions}

Acid survival experiments were performed in DMG. Strains were grown as described above in the presence or absence of $6.8 \%$ tryptone, or peptone, or casamino acids. The 10403S WT

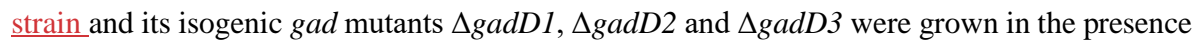
or absence of $6.8 \%, 3.6 \%, 10 \%$ tryptone, peptone and casamino acids respectively. Different concentrations were used depending on the gad mutants and to achieve measurable rate of death at similar $\mathrm{pH}$. To achieve death of cells at measurable rate, the $\mathrm{pH}$ of the overnight cultures was adjusted to 2.4 and 2.5 depending on the experiments performed and challenged with $\mathrm{HCl}(1 \mathrm{M})$. Samples were obtained prior to the $\mathrm{pH}$ adjustment and thereafter every $20 \mathrm{~min}$ 
up to 60 min decimal serial dilutions were prepared from those samples and plated onto BHI agar. Subsequently, plates were incubated at $37^{\circ} \mathrm{C}$ overnight, and subsequently, colonies were counted to assess survival under lethal acidic conditions. All experiments were performed in triplicate.

\section{GABase assay}

A commercial preparation known as GABase was used to determine the intracellular $\left(\mathrm{GABA}_{\mathrm{i}}\right)$ and extracellular $\left(\mathrm{GABA}_{\mathrm{e}}\right)$ concentrations of GABA. GABA was quantified as described by O'Byrne et al., (2011), while $\mathrm{GABA}_{\mathrm{e}}$ was quantified according to the method of Tsukatani et al., (2011) as modified by Karatzas et al. (2010). The pH of the cultures was adjusted to 4.2 to quantify the levels of $\mathrm{GABA}_{i}$ and $\mathrm{GABA}_{\mathrm{e}}$. This $\mathrm{pH}$ was chosen as it does not cause any cell death, which is essential when activity of a cellular system is measured, while it is low enough to ensure GAD system activation (Karatzas et al., 2012).

The GABase reaction was monitored by measurement of the absorbance at $340 \mathrm{~nm}$ every $2 \mathrm{~min}$ for $3 \mathrm{~h}$ at $37^{\circ} \mathrm{C}$ using a Sunrise spectrophotometer (Tecan, Mannedorf, Switzerland) operated by Magellan software (Tecan, Mannedorf, Switzerland). All reagents used for the GABase assay were obtained from Sigma-Aldrich (Steinheim, Germany).

\section{Real-time PCR determination of gad gene expression}

Transcription of the gad genes (gadD1, gadD2 and gadD3) was assessed as described previously by Karatzas et al., (2010) by real time reverse transcription-PCR (RT-PCR). Transcription of the antiporter-encoding genes ( $\operatorname{gadT1}$ and $\operatorname{gadT2}$ ) was not assessed because their transcription is similar to the corresponding glutamate decarboxylases ( $\mathrm{gadD} 1$ and gadD2 respectively) that belong to the same operon, as shown previously (Karatzas et al., 2012). Efficiencies of the primer pairs gadD1F-gadD1R, gadD2F-gadD2R, gadD3F-gadD3R and 
16SF-16SR were 2.12, 2.09, 2.03 and 2.27 respectively (Karatzas et al., 2010). Overnight cultures of 10403S WT were grown for $\sim 24 \mathrm{~h}$ in DMG in the presence or absence of $6.8 \%$ tryptone or peptone or casamino acids. Samples were taken at $28 \mathrm{~h}$ and relative expression of the data was calculated as a ratio between expression of each of the target genes and that of 16S rRNA which was used as the reference gene for each cDNA sample. The advanced relative quantification settings of the Light Cycler 480 SW 1.5.1 software programme were used, with PCR efficiency as described previously (Karatzas et al., 2010). Relative expression of each gene was calculated by dividing the values obtained for this gene with those for the 16S rRNA gene. Subsequently the relative expression values in the presence of either peptone, tryptone or casamino acids in DMG were compared to the controls without those compounds and fold changes were calculated.

\section{RESULTS}

\section{gad genes in $10403 S$ play no role in survival under acidic conditions in DMG}

We previously showed that glutamate supplementation of DM had no effect on survival of We previously showed that glutamate supplementation of DM had no effect on survival of We previously showed that glutamate supplementation of DM had no effect on survival of We previously showed that glutamate supplementation of DM had no effect on survival of We previously showed that glutamate supplementation of DM had no effect on survival of We previously showed that glutamate supplementation of DM had no effect on survival of

\section{Discovery of compounds resulting in $G A D_{e}$ activity and $G A B A_{e}$ export}

DMG was supplemented with different compounds or mixes of compounds and following the DMG was supplemented with different compounds or mixes of compounds and following the 
DMG was supplemented with different compounds or mixes of compounds and following the growth of L. monocytogenes 10403S, GADe activity was assessed through $\mathrm{GABA}_{\mathrm{e}}$ measurements to identify potential GAD activators (Fig. $\underline{1}$ ).

None of the individual compounds or mixes of compounds tested resulted in major $\mathrm{GAD}_{\mathrm{e}}$ activity, with the exception of an array of undefined mixes of amino acids and/or peptides such as casamino acids, tryptone and peptone. The supplementation of DMG with these mixes resulted in significant levels of $\mathrm{GABA}_{\mathrm{e}}(\mathbf{F i g} . \underline{\mathbf{1 A}})$. All these experiments were also performed under anoxic conditions

(Fig. and similarly, under these conditions the presence of casamino acids, tryptone and peptone activated the $\mathrm{GAD}_{\mathrm{e}}$ system. It seems that casamino acids resulted in higher levels of $\mathrm{GABA}_{\mathrm{e}}$ compared to other compounds tested. Under anoxic conditions, some levels of $\mathrm{GABA}_{\mathrm{e}}$ were observed in presence of lactic acid (Fig. $\left.1 \mathbf{B}\right)$.

\section{Acid resistance of $10403 S$ WT as a result of GAD system activation}

\section{Strain}

10403S WT grown overnight in $6.8 \%$ peptone or tryptone or casamino acids was challenged 10403S WT grown overnight in $6.8 \%$ peptone or tryptone or casamino acids was challenged with $\mathrm{HCl}$ at $2.4 \mathrm{pH}$. We settled doing experiments with one of the three concentrations used above $(6.8 \mathrm{mM})$ to allow comparisons between the different mixtures of compounds and because this concentration provided a clear difference with the negative control. In all cases, 10403S WT grown in DMG with peptone, tryptone and casamino acids was more resistant under acidic conditions compared to DMG alone (Fig. 2A).

During acid challenge for 40 min, cells of 10403S WT in DMG supplemented with peptone were the most resistant while those in DMG with tryptone or casamino acids were more 
sensitive, but still more resistant than those in DMG alone. Interestingly, during the course of the experiment, inactivation rate of cells in DMG with peptone or DMG with tryptone increased while that of cells in DMG with casamino acids decreased, at 60 min the latter had higher numbers than those grown in peptone and tryptone (Fig. $\underline{2} \mathrm{~A})$.

$\mathrm{GABA}_{e}$ measurements were performed in the presence of $6.8 \%$ of each one of these mixtures in $\mathrm{pH}$ 4.2. In the presence of these compounds, $\mathrm{GABA}_{\mathrm{e}}$ levels increased $(\mathrm{P}<0.05$, paired $\mathrm{t}$-test) steadily within the $60 \mathrm{~min}$ of the experiment while no GABA was observed in DMG. Tryptone and peptone similarly resulted in the highest levels of $\mathrm{GABA}_{\mathrm{e}}$ reaching close to $10 \mathrm{mM}$ after $60 \mathrm{~min}$ whereas casamino acids resulted in intermediate levels of $\mathrm{GABA}_{\mathrm{e}}$ close to $4 \mathrm{mM}$ (Fig. 2B). As expected, the non-supplemented control did not produce $\mathrm{GABA}_{\mathrm{e}}$ in response to acidification. Furthermore, upon supplementation with these three mixes of compounds we also observed statistically significant $(\mathrm{P}<0.05$, paired $t$-test $)$ higher levels of $\mathrm{GABA}_{i}$ production in comparison to the DMG control (Fig. 2 C).

\section{The role of $G A D$ genes in $G A D_{e}$ and $G A D_{i}$ activation and survival under acidic conditions}

Acid survival was assessed in DMG with supplementation of $3.6 \%, 6.8 \%$ and $10 \%$ peptone, Acid survival was assessed in DMG with supplementation of $3.6 \%, 6.8 \%$ and $10 \%$ peptone, Acid survival was assessed in DMG with supplementation of 3.6\%, 6.8\% and $10 \%$ peptone, Acid survival was assessed in DMG with supplementation of $3.6 \%, 6.8 \%$ and $10 \%$ peptone, tryptone and casamino acids individually respectively. In the presence of peptone, WT and its three gad mutants showed increased resistance compared to DMG alone (Fig. 3A). WT, $\triangle g a d D 1$ and $\triangle g a d D 3$ had very similar increased levels of $\mathrm{GABA}_{\mathrm{e}}$ in presence of peptone (Fig. $\underline{3} \mathbf{B})$. The $\Delta g a d D 2$ was the most sensitive strain either in the presence or absence of peptone suggesting that GadD2 is the most important component of the GAD 
251 system for survival (Fig. $\underline{3}$ A) and this coincides with no detectable levels of $\mathrm{GABA}_{\mathrm{e}}$ being

252 found in the $\Delta$ gadD2 (Fig. $\underline{3} \mathbf{B}$ ) either in presence or absence of peptone.

253 In the presence of tryptone, WT and all its gad mutants showed similar increased resistance compared to those ones without tryptone (Fig. $\underline{4} \mathbf{A})$. This coincides with increased levels of $\mathrm{GABA}_{\mathrm{e}}$, however $\triangle \mathrm{gadD} 2$ did not produce $\mathrm{GABA}_{\mathrm{e}}$ compared to other strains even in presence of tryptone (Fig. $\underline{4} \mathbf{B})$. If we follow the trend, we find that $\Delta g a d D 2$ with and without tryptone is the most sensitive during survival suggesting that GadD2 is important under acidic conditions.

The $\Delta g a d D 2$ was the most sensitive strain whereas WT, $\Delta$ gadD1, $\Delta g a d D 3$ were the most resistant in the presence of casamino acids. Although the presence of casamino

\section{S WT gad gene transcription}

Overall transcription of all GAD decarboxylases was low at basal conditions in DMG with Overall

transcription of all GAD decarboxylases was low at basal conditions in DMG with gadD3

The presence of these mixtures resulted in gadD2 having the highest transcription levels, followed by gadD3 while gadD1 had the lowest transcription. Remarkably, the supplementation with these three mixtures did not result in a significant change in the transcription of $\operatorname{gadD1}$ (Fig. ㅁA) and $\operatorname{gadD3}$ (Fig. 무) but only in that of gadD2. From all three mixtures, peptone had the strongest effect on gadD2 transcription followed by casamino acids and tryptone (Fig. $\underline{6} \mathbf{B})$. 
The GAD system plays an important role in L. monocytogenes under acidic conditions and it functions by converting extracellular glutamate to GABA, a reaction that removes protons from the intracellular milieu and helps to maintain pH homeostasis (Karatzas et al., 2010; O’Byrne and Karatzas 2008; Waterman and Small 2003). Although work has increased our knowledge on the function of the GAD system in this organism and other Gram-positive bacteria, the effects of environmental conditions and signals on its expression and function are still poorly understood. The only known environmental conditions known to result in increased transcription of the GAD system in this organism are growth in rich media under stationary phase (Cotter et al., 2001a; Cotter et al., 2001b; Penfound et al., 1999), anoxic conditions (Feehily et al., 2013; Jydegaard-Axelsen et al., 2004) and gastric fluid (Cotter et al., 2001a). No other conditions are currently known to activate the system and even the first condition is vague as the specific compounds, other than glutamate, that lead to activation in the rich media are unknown.

In our study, we used the chemically defined medium (Amezaga et al., 1995), which was previously shown to prevent functioning of the $\mathrm{GAD}_{\mathrm{e}}$ system in this organism, even upon supplementation with glutamate under acidic conditions (Karatzas et al., 2010). This suggests that unknown compounds are essential for GAD activity in L. monocytogenes (Karatzas et al., 2010) and we attempt to identify these compounds in this study. We used this DMG medium as a basis for our screening experiments since L. monocytogenes is unable to export GABA in this medium. Supplementation of this medium with various compounds could give an indication of which compounds are required for GAD system upregulation and/or function. Since we also planned to see which part of the GAD system is affected by the supplemented compound(s) we initially assessed if the removal of each one of the decarboxylases in 10403S had an effect on survival in DMG. No difference was observed (Fig. 1 $\underline{\text { S}}$ ) confirming that each of the GAD system components is completely inactive in this medium. Subsequently, a variety 
of different compounds was tested for their ability to activate the GAD system of $L$. monocytogenes. None of the individual compounds resulted in increased GAD activity however, supplementation of DMG while all three crude mixtures of aminoacids and/or peptides namely, tryptone, peptone and casamino acids resulted in significant $\mathrm{GAD}_{\mathrm{e}}$ activity (Fig. 1A). As anoxic conditions have been shown to activate expression of the GAD system in rich media (Jydegaard-Axelsen et al., 2004) we repeated the above experiments under anoxic conditions. Similar results were obtained in oxic conditions however, levels of GABA looked higher (Fig. 1B). These mixes also activated the $\mathrm{GAD}_{\mathrm{i}}$ system (Fig. $1 \mathbf{C}$ ) and together with the increased $\mathrm{GAD}_{\mathrm{e}}$ activity, the overall increased GAD activity resulted in increased acid resistance (Fig. $\underline{\mathbf{1 A}})$. It should be stated that this increased resistance might also be the result of other mechanisms however, most probably it is majorly the result of GAD system activation since this is the most dominant acid resistance mechanism.

It could be suggested that the above observed GAD system activation and increased acid resistance is the result of osmotic effects. To clarify this, we could compare the osmotic pressure in DMG supplemented with $0.5 \mathrm{M} \mathrm{NaCl}$ with DMG supplemented with the amino acid and peptide mixes. However, it is difficult to accurately calculate the levels of osmotic pressure in the DMG supplemented with the amino acid and peptide mixes since they are crude and not defined. However, it is known from chemical analyses of these mixes that their average molecular weight is above 250 Daltons (>250 g/mol). Based on this value a 2, 3.6, 6.8 and $10 \%$ solution is equivalent to $0.08,0.14,0.27,0.40 \mathrm{M}$ respectively. Therefore, since $0.5 \mathrm{M} \mathrm{NaCl}$ has absolutely no effect on GABA export and given that the molarity of all these mixtures is significantly lower than $0.5 \mathrm{M}$, we could conclude that the effects observed were not related to osmotic pressure. 
Interestingly, all three activators identified here were crude mixtures of amino acids and peptides. Casamino acids are a mixture of free amino acids and some very small peptides resulting from the acid hydrolysis of casein (Mueller and Johnson, 1941) whereas tryptone is an assortment of peptides formed by the digestion of casein by the protease trypsin (Fraser and Powell, 1950). Peptone is derived from animal milk or meat digested by proteolysis. It contains peptides, salts, vitamins and many other biological compounds (Payne, 1976). Peptone and tryptone are rich in tryptophan whereas casamino acids lack tryptophan because of acid treatment during casein hydrolysis.

As seen in Fig. $\underline{2} \mathbf{B}$ and by comparing the overall effect shown in Fig. $\underline{3} \mathbf{B}, \underline{4} \mathbf{B}$ and $\underline{5} \mathbf{B}$, supplementation with peptone and tryptone resulted in similarly high levels of $\mathrm{GAD}_{\mathrm{e}}$ activity. Furthermore, the effect of casamino acids on $\mathrm{GAD}_{\mathrm{e}}$ activity seemed to be weaker compared to the effect of the above compounds (Fig. 2B) although the effect on $\mathrm{GABA}_{\mathrm{i}}$ was similar to tryptone and peptone (Fig. 2C). We speculate that some higher peptides contained in these compounds but not in casamino acids might be activating the $\mathrm{GAD}_{\mathrm{e}}$ system. However, it should be stated that under anoxic conditions casamino acids seem to have a higher effect on $\mathrm{GAD}_{\mathrm{e}}$ activity than the other two compounds (Fig. 1). Although supplementation of DMG with these compounds increases $\mathrm{GABA}_{e}, \mathrm{GABA}_{\mathrm{i}}$ levels and acid resistance, the overall $\mathrm{GABA}_{\mathrm{e}}$ levels achieved by each of these mixes of compounds did not completely correspond to the acid resistance. For example, although supplementation with peptone and tryptone resulted in similar GABAe levels (Fig. $\underline{2}$ B), peptone conferred a higher acid resistance than tryptone (Fig. 2A). Furthermore, although supplementation with casamino acids provided a much weaker $\mathrm{GAD}_{\mathrm{e}}$ activity compared to tryptone and peptone (Fig. $\left.2 \mathbf{B}\right)$, it conferred the highest acid resistance (Fig. 2AA). As stated before, other acid resistance and survival mechanisms might be affected by the supplementation by these compounds. However, it should be noted that the general trend in $\mathrm{GAD}_{\mathrm{e}}$ activity corresponded with the trends in survival. It 
could be seen that upon supplementation with casamino acids, deployment of both $\mathrm{GAD}_{\mathrm{e}}$ and acid resistance was delayed as both curves seem to have an upward trend during the course of the experiment (Fig. 2 A, B). In contrast, supplementation with tryptone and peptone resulted in a rapid deployment of $\mathrm{GAD}_{\mathrm{e}}\left(\sim 70 \%\right.$ of $\mathrm{GABA}_{\mathrm{e}}$ is produced within the first 20 min) which corresponded well with a higher acid resistance in the initial stages (Fig. 2A, B). As the experiment progressed, GAD e activity of cultures supplemented with tryptone or peptone seemed to reduce, which correlated well with a reduction in acid resistance at later stages (Fig. $\underline{2} \mathbf{A}, \mathbf{B})$. The above suggests that the time of the $\mathrm{GAD}_{\mathrm{e}}$ deployment is critical for survival. Furthermore, it should be stated that acid survival at $60 \mathrm{~min}$ (Fig.3A) correlated well with the $\mathrm{GABA}_{i}$ levels (Fig. 2 C), with casamino acids having the highest effect followed by peptone and tryptone. However, $\mathrm{GABA}_{\mathrm{i}}$ levels are not only the result of $\mathrm{GAD}_{\mathrm{i}}$ activity but are also affected by GABA catabolism through the GABA shunt (Feehily et al.,2013). It should be stated though that, high levels of $\mathrm{GABA}_{\mathrm{i}}$ are possibly associated with higher $\mathrm{GAD}_{\mathrm{i}}$ activity as GABA catabolism might not be affected significantly, but this is an area that requires further work. Overall it could be said that peptone and tryptone resulted in higher $\mathrm{GAD}_{\mathrm{e}}$ activity, while casamino acids most probably resulted in higher $\mathrm{GAD}_{\mathrm{i}}$ activity.

Similarly, in previous work casamino acids enhanced prolonged survival of Streptococcus lactis by supplying amino acids and minimizing break down of essential components needed for survival (Thomas and Batt, 1968). However, we are not aware if this strain possessed a GAD system. Another study showed that various E. coli strains and several other bacteria had less acidic shock in Tryptone Soy Broth when supplemented with casamino acids, however there was decreased survival in presence of glutamate alone (Park and Diez-Gonzalez, 2004). These authors have suggested that additional amino acids might enhance the glutamatedependent acid resistance, which agrees with what we demonstrate here. Recent work has also demonstrated that the presence of amino acids enhanced the ability of L. monocytogenes to 
grow under mild acidic conditions although no explanation was offered for this (Muchaamba et al., 2019).

Furthermore, we investigated the effect of $6.8 \%$ tryptone $3.6 \%$ peptone and $10 \%$ casamino acids on strains carrying deletions of the GAD decarboxylase genes. We used different concentrations of these compounds in order to obtain measurable levels of inactivation during these survival experiments. The results showed that the $\Delta g a d D 2$ was the most sensitive either in the presence or absence of peptone or tryptone (Fig. $\underline{3} \mathbf{A}, \underline{4} \mathbf{A})$. Similarly, in presence of casamino acids $\Delta g a d D 2$ was the most sensitive (Fig. $\underline{\mathbf{5}} \mathbf{A})$. Even in presence of either of those compounds $\Delta$ gadD2 exported less $\mathrm{GABA}_{\mathrm{e}}$ than the other mutants and the WT (Fig. $\underline{3} \mathbf{B}, \underline{4} \mathbf{B}$, 5B). The results suggest that GadD2 is the GAD component most affected by supplementation. This is expected as GadD2 is the main component of the GADe system that is responsible for GABA export (Cotter et al., 2001a; Cotter et al., 2005) and and highly important for survival under acidic conditions (Cotter et al., 2001a). WT, $\Delta$ gadD1 and $\triangle g a d D 2$ were able to export $\mathrm{GABA}_{\mathrm{e}}$ in the presence of peptone, tryptone, or casamino acids which also suggests that these compounds might activate the GAD system. This might be the reason for the ability of the strains to export GABAe.

Subsequently, to identify how these compounds activate the GAD system, we performed RTqPCR to quantify the transcription of $\operatorname{gadD1}$, gadD2 and $\operatorname{gadD} 3$ that encode the three different decarboxylases of the GAD system. We looked at transcription during stationary phase as it is well-known that the GAD system is mainly expressed at this stage of growth. Furthermore, we have shown previously that $L$. monocytogenes does not respond to acidic conditions by significant increase in the transcription of the GAD system genes gadD1, gadD2 and gadD3 (Karatzas et al., 2010; Karatzas et al., 2012). It seems that the GAD system needs to be deployed prior to acidification and therefore we just assessed transcription at stationary phase and not following an acid drop. Furthermore, we did not quantify the transcription of 
antiporters $\operatorname{gadT1}$ or $\operatorname{gadT2}$ as it has been previously confirmed that they follow the transcription of their corresponding GAD decarboxylases (gadD1 and gadD2) with which they belong to the same operon (Karatzas et al., 2012).

Previously it has been shown that GadD2 is the most important part of the GAD system under acidic stress (Cotter et al., 2001a; Cotter et al., 2005). In 10403S WT (Fig. $\underline{6}$ B) peptone had the strongest effect on gadD2 transcription followed by casamino acids and tryptone (Fig. $\underline{6} \mathbf{B})$. Although previously GadD3 was identified as a part of the $\mathrm{GAD}_{\mathrm{i}}$ system, it did not seem to be upregulated by any of the compounds used here and it did not seem to affect survival in any of the experiments. It might be possible that GadD3 plays a role under different conditions or activated by other environmental conditions.

Up to now there is no previous work showing environmental conditions or signals that affect expression of the GAD system in L. monocytogenes and most other microorganisms with the exception of E. coli. Our research contributes to our understanding of the activation of the GAD system and the acid resistance in this organism. We show that casamino acids, peptone and tryptone are major GAD system activators resulting in upregulation of the transcription of gadD2, which is the most important component of the GAD system. This research would be important to scientists working on the acid resistance of L. monocytogenes and other organisms. It can also help us predict the behaviour of L. monocytogenes in acidic foods and contribute in the development of strategies to manipulate the acid resistance of L. monocytogenes and possibly other pathogens by restricting the availability of these activators. This could lead to the elimination of these pathogens from food and therefore contribute in the reduction of foodborne infections.

\section{Acknowledgements.}


We would like to thank all colleagues at the Microbiology research team, Food and Nutritional Sciences, University of Reading. The help of Marcia Boura in reviewing the manuscript and the help and support of our deceased colleague Dr. Bernard Mackey has been invaluable. This work was partly supported by a Marie Curie European Reintegration Grant (grant ERG 265154), a Science Foundation Ireland Starting Investigator Research Grant (SIRG; grant 09/SIRG/B1570) awarded to K. A. Karatzas, and funds from the University of Reading.

\section{REFERENCES}

Amezaga, M.-R., Davidson, I., McLaggan, D., Verheul, A., Abee, T., Booth, I.R., 1995. The role of peptide metabolism in the growth of Listeria monocytogenes. Microbiol. 141,4149. doi.org/10.1099/00221287-141-1-41.

Capitani, G., Biase, D De., Aurizi, C., Gut, H., Bossa, F., Gru, MG., Ch, È., 2003. Crystal structure and functional analysis of Escherichia coli glutamate decarboxylase. EMBO J. 22, 4027-4037. doi:10.1093/emboj/cdg403.

Cole, S.T., Brosch, R., Parkhill, J., Garnier, T., Churcher, C., Harris, D., Gordon, S.V., Eiglmeier, K., Gas, S., Barry, C.E., Tekaia, F., Badcock, K., Basham, D., Brown, D., Chillingworth, T., Connor, R., Davies, R., Devlin, K., Feltwell, T., Gentles, S., Hamlin, N, Holroyd, S., Hornsb,y T., Jagels, K., Krogh, a., McLean, J., Moule, S., Murphy, L., Oliver, K., Osborne, J., Quail, M a., Rajandream, M a., Rogers, J., Rutter, S., Seeger, K., Skelton, J., Squares, R., Squares, S., Sulston, J.E., Taylor, K., Whitehead, S., Barrell, B.G., 1998. Deciphering the biology of Mycobacterium tuberculosis from the complete genome sequence. Nature. 393,537-544. doi:10.1038/31159.

Cotter, P.D., Gahan, C.G., Hill C., 2001a. A glutamate decarboxylase system protects Listeria 
monocytogenes in gastric fluid. Mol. Microbiol 40, 465-475. doi.org/10.1046/j.13652958.2001.02398.x.

Cotter, PD., O’Reilly, K., Hill, C., 2001b. Role of the glutamate decarboxylase acid resistance system in the survival of Listeria monocytogenes LO28 in low pH foods. J. Food. Prot. 64,1362-1368. doi: 10.4315/0362-028x-64.9.1362.

Cotter, P.D., Ryan, S., Gahan, C.G.M., Hill, C., 2005. Presence of GadD1 glutamate decarboxylase in selected Listeria monocytogenes strains is associated with an ability to grow at low pH. Appl. Environ. Microbiol. 71,2832-2839. doi: 10.1128/AEM. 71.6.2832-2839.

Damiano, M. A., Bastianelli, D., Al Dahouk, S., Köhler, S., Cloeckaert, A., De Biase, D., Occhialini, A. 2014. Glutamate decarboxylase-dependent acid resistance in Brucella spp.: distribution and contribution to fitness under extreme acid conditions. Appl. Environ. Microbiol. 81(2):578-86. https://doi.org/10.1128/AEM.02928-14

European Food Safety Authority and European Centre for Disease Prevention and Control $\underline{\text { EFSA and ECDC). 201․ The European Union }}$ summary report on trends and sources of zoonoses, zoonotic agents and food-borne outbreaks in 2017

\begin{tabular}{llr} 
EFSA & Journal, & 16 \\
\hline
\end{tabular}

(12). doi.org/10.2903/j.efsa.2018.5500.

Feehily, C., Finnerty, A., Casey, P.G., Hill, C., Gahan, C.G.M., O’Byrne, C.P., Karatzas, K.A.G., 2014. Divergent evolution of the activity and regulation of the glutamate decarboxylase systems in Listeria monocytogenes EGD-e and 10403S: Roles in virulence and acid tolerance. PloS one 9,e112649. doi:10.1371/journal.pone.0112649.

Feehily, C., O’Byrne, C.P., Karatzas, K.-A.G., 2013. Functional $\gamma$-aminobutyrate shunt in Listeria monocytogenes: role in acid tolerance and succinate biosynthesis. Appl. 
Environ. Microbiol. 79,74-80. doi:10.1128/AEM.02184-12.

Foster, JW., 2004. Escherichia coli acid resistance: tales of an amateur acidophile. Nature Rev. Microbiol. 2,898-907. doi: 10.1038/nrmicro1021.

Hersh, B.M., Farooq, F.T., Barstad, D.N., Blankenhorn, D.L., Hersh, BM., Farooq, F.T., Slonczewski, J.L., 1996. A glutamate-dependent acid resistance gene in Escherichia coli . J Bacteriol. 178,3978-81. doi: 10.1128/jb.178.13.3978-3981.

Jydegaard-Axelsen, A.-M., Høiby, P.E., Holmstrøm, K., Russell, N., Knøchel, S., 2004. CO2and anaerobiosis-induced changes in physiology and gene expression of different Listeria monocytogenes strains. Appl. Environ. Microbiol. 70,4111-4117. doi: 10.1128/AEM.70.7.4111-4117.2004

Karatzas, K.-AG., Brennan, O., Heavin, S., Morrissey, J., O’Byrne, C.P., 2010. Intracellular accumulation of high levels of gamma-aminobutyrate by Listeria monocytogenes 10403S in response to low $\mathrm{pH}$ : uncoupling of gamma-aminobutyrate synthesis from efflux in a chemically defined medium. Appl. Environ. Microbiol. 76,3529-37. doi:10.1128/AEM.03063-09.

Karatzas, K.-A.G., Suur, L., O'Byrne, C.P., 2012. Characterization of the intracellular glutamate decarboxylase system: analysis of its function, transcription, and role in the acid resistance of various strains of Listeria monocytogenes. Appl. Environ. Microbiol. 78,3571-9. doi:10.1128/AEM.00227-12.

Muchaamba, F., Eshwar, A.K., Stevens, M.J.A., von Ah, U., Tasara T., 2019. Variable carbon source utilization, stress resistance, and virulence profiles among Listeria monocytogenes strains responsible for listeriosis outbreaks in Switzerland. Frontiers Microbiol. 10, 957. doi:10.3389/fmicb.2019.00957.

Mueller, J.H., Johnson, E. R., 1941. Acid hydrolysates of casein to replace peptone in the preparation of bacteriological media. J. Immunol. 40,33-38. 
Fraser, D., Powell, E., 1950. The kinetics of trypsin digestion. J. Biol. Chem. 187,803-820.

O’Byrne, C.P., Karatzas, K.A.G., 2008. Chapter 5. The Role of Sigma B $(\sigma B)$ in the stress adaptations of Listeria monocytogenes: Overlaps between stress adaptation and virulence. Adv. Appl. Microbiol. 65,115-140. doi: 10.1016/S0065-2164(08)00605-9

O'Byrne, C.P. , Feehily, C. , Ham, R. and Karatzas, K.A.G., 2011. A modified rapid enzymatic microtiter plate assay for the quantification of intracellular $\gamma$-aminobutyric acid and succinate semialdehyde in bacterial cells. J. Microbiol. Meth., 84 (1), 137-139. doi: https://doi.org/10.1016/j.mimet.2010.10.017.

Park, G.W., Diez-Gonzalez, F., 2004. A novel glutamate-dependent acid resistance among strains belonging to the proteeae tribe of enterobacteriaceae. FEMS Microbiol. Lett. 237,303-9. doi: 10.1016/j.femsle.2004.06.050.

Payne, J.W., 1976. Peptides and Micro-Organisms. Adv. Microb. Physiol.13,55-113.

Penfound, T.A., Elliott, J.F., Foster, J.W., Smith, D., 1999. Control of acid resistance in Escherichia coli. J Bacteriol . 181,3525-35.

Shelp, B.J., Bown, A.W., Mclean, M.D., 1999. Metabolism and functions of gammaaminobutyric acid. Trends Plant Sci. 4, 446-452. doi.org/10.1016/S13601385(99)01486-7.

Su, M.S., Schlicht, S., Gänzle, M.G., 2011. Contribution of glutamate decarboxylase in Lactobacillus reuteri to acid resistance and persistence in sourdough fermentation. Microb. Cell Fact. 10,S8. doi:10.1186/1475-2859-10-S1-S8.

Thomas, T.D., Batt, RD., 1968. Survival of Streptococcus lactis in starvation conditions J. gen. Microbiol. 50,367-382. doi: 10.1099/00221287-50-3-367.

Tsukatani, T., Higuchi, T., Matsumoto, K., 2005. Enzyme-based microtiter plate assay for $\gamma$ aminobutyric acid: application to the screening of $\gamma$-aminobutyric acid-producing lactic acid bacteria. Anal. Chim. Acta, 540, 293-297 


\begin{tabular}{|c|c|c|c|}
\hline Strain & Description & Nature of mutation & Source reference \\
\hline $10403 \mathrm{~S}$ & Serotype $1 / 2 \mathrm{a}$, wild type & & (Karatzas et al.,2010) \\
\hline$\Delta \operatorname{gadD} 1$ & $10403 \mathrm{~S}$ isogenic gadD1 mutant & In-frame deletion & (Feehily et al., 2014) \\
\hline$\triangle \operatorname{gadD} 2$ & $10403 \mathrm{~S}$ isogenic gadD2 mutant & In-frame deletion & (Feehily et al., 2014) \\
\hline$\Delta \operatorname{gadD} 3$ & $10403 \mathrm{~S}$ isogenic gadD3 mutant & In-frame deletion & (Feehily et al., 2014) \\
\hline
\end{tabular}

538

542 Fig. 1. Survival after acid challenge stationary phase cultures of $10403 \mathrm{~S}$ WT and its isogenic

Waterman, S.R., Small, P.L.C., 2003. The glutamate-dependent acid resistance system of Escherichia coli and Shigella flexneri is inhibited in vitro by L-trans-pyrrolidine-2,4dicarboxylic acid. FEMS Microbiol. Lett. 224,119-125. doi:10.1016/S03781097(03)00427-0.

TABLES

Table 1. Strains used in this study

\section{LEGEND TO FIGURES}

gad mutants in DMG. Cultures were grown at $37^{\circ} \mathrm{C}$ with shaking until stationary phase in DMG 
Fig. 1. Cells of 10403S WT grown until stationary phase in DMG in the presence of $0.5 \mathrm{mg} / \mathrm{ml}$ of sodium chloride, $0.5 \mathrm{mg} / \mathrm{ml}$ maleic acid, $0.5 \mathrm{mg} / \mathrm{ml}$ succinic acid, $0.5 \mathrm{mg} / \mathrm{ml}$ acetic acid, 0.5 $\mathrm{mg} / \mathrm{ml}$ lactic acid, $0.1 \%$ sodium deoxycholate, $5 \%$ oxgall, $5 \%$ bile salts, $2 \%, 10 \%$ casamino acids $(\mathrm{CA}), 3.6 \%$ peptone and $6.8 \%$ tryptone. Following growth, the $\mathrm{pH}$ was adjusted to $\mathrm{pH} 4$ the extracellular GABA $\left(\mathrm{GABA}_{\mathrm{e}}\right)$ was measured under (A) oxic and (B) anoxic conditions in DMG. Bars represent an average of measurements performed in triplicate, and error bars represent the standard deviation. D.L. denotes the detecion limit of the GABase method.

Fig. 2. (A) Cells of 10403S WT grown under oxic conditions in DMG alone (grey circles) or in DMG with 6.8\% tryptone (grey diamonds), peptone (black squares), casamino acids (C.A.; black triangles) grown under oxic conditions until stationary phase ( 24 h). Subsequently, cultures were acid challenged at $\mathrm{pH} 2.4$ with the addition of $1 \mathrm{M} \mathrm{HCl}$. Monitoring $\mathrm{GABA}_{\mathrm{e}}(\mathrm{B})$ was conducted for $0,20,40$ and $60 \mathrm{~min}$ and $\mathrm{GABA}_{\mathrm{i}}(\mathrm{C})$ for $60 \mathrm{~min}$. $\mathrm{GABA}_{\mathrm{e}}$ and $\mathrm{GABA}_{\mathrm{i}}$ were assessed following adjustment of the $\mathrm{pH}$ of overnight cultures of 10403S WT at pH 4.2 grown until stationary phase in DMG and DMG with $6.8 \%$ tryptone, peptone and casamino acids (C.A.). Markers represent an average of measurements performed in triplicate, and error bars represent the standard deviation. D.L denotes detection limit. Asterisk represents statistical significant difference with the control cultures grown in DMG $(\mathrm{P}<0.05)$.

Fig. 3. (A) Cells of 10403S WT (white boxes) and its gad mutants $\Delta$ gadDl (white triangles), $\Delta g a d D 2$ (white circles) and $\Delta g a d D 3$ (white diamonds) grown in DMG alone or in DMG with $3.6 \%$ peptone, with WT (black boxes) and its gad mutants $\Delta$ gadD1 (black triangles), $\Delta$ gadD2 (black circles) and $\triangle$ gadD3 (black diamonds) grown under oxic conditions until 
stationary phase $(\sim 24 \mathrm{~h})$. Subsequently, cultures were acid challenged at $\mathrm{pH} 2.5$ with the addition of $1 \mathrm{M} \mathrm{HCl}$. Monitoring of $\mathrm{GABA}_{\mathrm{e}}(\mathrm{B})$ was conducted for $0,20,40$ and $60 \mathrm{~min}$ and were assessed following adjustment of the $\mathrm{pH}$ of overnight cultures of 10403S WT and its gad mutants at $\mathrm{pH} 4.2$ grown until stationary phase in DMG and in DMG with $3.6 \%$ peptone. Markers represent an average of measurements performed in triplicate, and error bars represent the standard deviation. D.L denotes detection limit.

Fig. 4. (A) Cells of 10403S WT (white boxes) and its gad mutants $\Delta$ gadDl (white triangles), $\Delta g a d D 2$ (white circles) and $\Delta g a d D 3$ (white diamonds) grown in DMG alone or in DMG with $6.8 \%$ tryptone, with WT (black boxes) and its gad mutants $\Delta$ gadD1 (black triangles), $\Delta$ gadD2 (black circles) and $\Delta g a d D 3$ (black diamonds) grown under oxic conditions until stationary phase $(\sim 24 \mathrm{~h})$. Subsequently, cultures were acid challenged at $\mathrm{pH} 2.5$ with the addition of $1 \mathrm{M} \mathrm{HCl}$. Monitoring of $\mathrm{GABA}_{\mathrm{e}}(\mathrm{B})$ was conducted for $0,20,40$ and $60 \mathrm{~min}$ and were assessed following adjustment of the $\mathrm{pH}$ of overnight cultures of 10403S WT and its gad mutants at $\mathrm{pH} 4.2$ grown until stationary phase in DMG and in DMG with $6.8 \%$ tryptone. Markers represent an average of measurements performed in triplicate, and error bars represent the standard deviation. D.L denotes detection limit.

Fig. ‥ (A) Cells of 10403S WT (white boxes) and its gad mutants $\Delta$ gadDl (white triangles), $\Delta g a d D 2$ (white circles) and $\Delta g a d D 3$ (white diamonds) grown in DMG alone or in DMG with $10 \%$ casamino_acids_(C.A.), with WT (black boxes) and its gad mutants $\Delta$ gadDl (black triangles), $\Delta g a d D 2$ (black circles) and $\Delta g a d D 3$ (black diamonds) grown under oxic conditions until stationary phase $(\sim 24 \mathrm{~h})$. Subsequently, cultures were acid challenged at $\mathrm{pH}$ 2.5 with the addition of $1 \mathrm{M} \mathrm{HCl}$. Monitoring of $\mathrm{GABA}_{\mathrm{e}}(\mathrm{B})$ was conducted for $0,20,40$ and $60 \mathrm{~min}$ and were assessed following adjustment of the $\mathrm{pH}$ of overnight cultures of 10403S WT 
and its gad mutants at $\mathrm{pH} 4.2$ grown until stationary phase in DMG and in DMG with $10 \%$ casaminoacids (C.A.). Markers represent an average of measurements performed in triplicate, and error bars represent the standard deviation. D.L denotes detection limit.

Fig. 6. Real-time PCR determination of transcription of gadD1, gadD2 and gadD3. Relative expression of each gene was calculated by comparing expression relative to that of 16S rRNA in 10403S WT cultures grown overnight until stationary phase in DMG or DMG supplemented with $6.8 \%$ tryptone, peptone or casamino_acids (C.A.). Markers represent an average of measurements performed in triplicate, and error bars represent Markers represent an average of measurements performed in triplicate, and error bars represent Markers represent an average of measurements performed in triplicate, and error bars represent standard deviations. Asterisks represent statistically significant difference in the expression of each gene in the supplemented media compared to that in DMG $(\mathrm{P}<0.05)$. Numbers above the bars indicate fold difference of the relative expression for each gene in DMG supplemented with either tryptone, peptone or casaminoacids (C.A.) compared to its expression in DMG without supplementation.

\section{$\underline{\text { Supplementary data }}$}

\section{LEGEND TO FIGURES}

Fig. 1. Survival after acid challenge stationary phase cultures of 10403S WT and its isogenic gad mutants in DMG. Cultures were grown at $37^{\circ} \mathrm{C}$ with shaking until stationary phase in DMG and subsequently, the medium was adjusted to $\mathrm{pH} 2.4$ with the addition of $\mathrm{HCl}$ and survival was assessed after 60 min through preparation of decimal dilutions and plating. Error bars 
619 represent standard deviation from triplicate observations. 